\title{
Evidence for Polaron Formation in High-Temperature Superconducting Cuprates: Experiment and Theory
}

\author{
Annette Bussmann-Holder · Hugo Keller
}

Received: 20 October 2008 / Accepted: 20 October 2008 / Published online: 6 November 2008

(C) Springer Science+Business Media, LLC 2008

\begin{abstract}
We provide compelling support for the key role played by polaron formation to the physics of cuprate superconductors, which is evidenced above the pseudogap temperature $T^{*}$, is the origin of the pseudogap phase itself and persists in the superconducting phase. Experimental and theoretical results are compared and show convincing agreement with each other.
\end{abstract}

Keywords High temperature superconductivity - Lattice effects · Polaron formation

\section{Introduction}

Early and more recent isotope effect experiments on the superconducting transition temperature $T_{c}$ in high-temperature superconducting cuprates (HTS) have shown that the isotope effect is strongly doping dependent in these systems and especially strongly enhanced as compared to the BCS value at the borderline to the antiferromagnetic state [1-4]. Even though these observations do not necessarily support phonon mediated superconductivity in the classical sense, further isotope effects on other quantities suggest that lattice involvement is essential to the physics of HTS. These are: isotope effects on $T^{*}[5,6]$, the penetration depth $\lambda$ [2],

\footnotetext{
A. Bussmann-Holder ( $\varangle)$

Max-Planck-Institut für Festkörperforschung, Heisenbergstr. 1,

70569 Stuttgart, Germany

e-mail: a.bussmann-holder@fkf.mpg.de

H. Keller

Physik-Institut der Universität Zürich, Winterthurerstr. 190,

8057 Zürich, Switzerland
}

the spin glass temperature $T_{\mathrm{SG}}$, the antiferromagnetic transition temperature $T_{N}$ [7], the average superconducting energy gap $\Delta[8]$, with all of them being interrelated and strongly doping dependent in analogy to the one of $T_{c}$. In addition, local lattice anomalies are present which drive the system into a dynamically inhomogeneous state emerging from charge-rich and charge-poor, but spin-rich, regions [912]. Furthermore, there is unambiguous evidence that the superconducting gap symmetry is much more complex than simple d-wave type, but consists of an $(s+d)$-wave admixture which differs between the crystallographic directions $a, b$, and $c$ [13-15].

Here we demonstrate that these unconventional properties can be consistently described by a multicomponent approach with strong polaron coupling, thus demonstrating that the original concept which led to the discovery of hightemperature superconductivity is indeed realized [16, 17]. In the following we first present the theoretical background and the results from our model. Then, we compare these to experimental data to be followed by conclusions.

\section{Theoretical Modeling}

Even though the physics of the HTS are rather complex we start from a simple Hamiltonian which captures the essential features and accounts for the interactions between charge and lattice degrees of freedom [18-20]:

$$
\begin{aligned}
H= & \sum_{i} \varepsilon(i) c_{i}^{+} c_{i}+\sum_{q, j} \hbar \omega_{q, j}^{(0)}\left(b_{q, j}^{+} b_{q, j}+\frac{1}{2}\right) \\
& -\sum_{i} c_{i}^{+} c_{i} \sum_{q, j} \hbar \omega_{q, j}^{(0)} \gamma_{i}(q)\left(b_{q}^{+}+b_{q}\right)
\end{aligned}
$$




$$
-\sum_{i, m} c_{m}^{+} c_{i} \sum_{q, j} \tilde{\gamma}_{i, m}(q)\left(b_{q, j}+b_{q, j}^{+}\right) .
$$

Here $c^{+}, c$ are site $i$ dependent electron creation and annihilation operators with energies $\varepsilon$, and $b^{+}, b$ are phonon creation and annihilation operators with momentum $q$ and branch $j$ dependent bare phonon energy $\omega_{q, j}^{(0)}$. The coupling between electron and lattice degrees of freedom is given by $\gamma$ and $\tilde{\gamma}$, respectively, where the former is the diagonal coupling and the latter the off-diagonal coupling, which we take as a fraction of the diagonal one. The Hamiltonian (1) can have a variety of consequences: (a) lattice instability; (b) charge density wave formation; (c) BCS type superconductivity; and (d) polaron formation. Since (a), (b), and (c) can be excluded experimentally, we concentrate in the following on (d). In order to decouple charge and lattice degrees of freedom, the Lang-Firsov canonical transformation [21] is applied, which is explicitly given by $\tilde{H}=e^{-S} H e^{S}$, with $S=\sum_{i, q} c_{i}^{+} c_{i}\left(b_{q}^{+}-b_{q}\right) \gamma_{i}(q)$. From this transformation exact relations for phonon and electron creation and annihilation operators are obtained:

$\tilde{c}_{i}=c_{i} \exp \left[\sum_{q} \gamma_{i}(q)\left(b_{q}^{+}-b_{q}\right)\right]$

$\tilde{c}_{i}^{+}=c_{i}^{+} \exp \left[-\sum_{q} \gamma_{i}(q)\left(b_{q}^{+}-b_{q}\right)\right]$

$\tilde{b}_{q}=b_{q}+\sum_{q} \gamma_{i}(q) c_{i}^{+} c_{i}$

$\tilde{b}_{q}^{i}=b_{q}^{+}+\sum_{q} \gamma_{i}(q) c_{i}^{+} c_{i}$.

First, we consider the consequences arising from (2a), from which we conclude that the electronic energy bands experience a substantial renormalization [18, 19, 22]. By using the LDA-derived band structure for the cuprates [23], i.e.,

$$
\begin{aligned}
\varepsilon(k)= & -2 t_{1}\left(\cos k_{x} a+\cos k_{y} b\right)+4 t_{2} \cos k_{x} a \cos k_{y} b \\
& +2 t_{3}\left(\cos 2 k_{x} a+\cos 2 k_{y} b\right) \\
& \mp t_{4}\left(\cos k_{x} a-\cos k_{y} b\right)^{2} / 4-\mu,
\end{aligned}
$$

with $t_{1}, t_{2}, t_{3}$ being nearest, second-nearest and third-nearest neighbor hopping integrals in the $\mathrm{CuO}_{2}$ planes, $t_{4}$ is the interlayer hopping between the $\mathrm{CuO}_{2}$ planes, and $\mu$ is the chemical potential which controls the band filling, the application of (2a) modifies (3) to

$$
\begin{aligned}
\tilde{\varepsilon}(k)= & -2 t_{1}\left(\cos k_{x} a+\cos k_{y} b\right)+4 \tilde{t}_{2} \cos k_{x} a \cos k_{y} b \\
& +2 t_{3}\left(\cos 2 k_{x} a+\cos 2 k_{y} b\right) \\
& \mp \tilde{t}_{4}\left(\cos k_{x} a-\cos k_{y} b\right)^{2} / 4-\mu-\Delta *(k) .
\end{aligned}
$$

Beside the rigid band shift $\Delta^{*}$, only two hopping integrals, namely the second-nearest neighbor and the interlayer hopping, are affected and are explicitly given by

$\tilde{t}_{2,4}=t_{2,4} \exp \left[\gamma^{2} \operatorname{coth} \frac{\hbar \omega_{q, j}^{(0)}}{2 k_{B} T}\right]$.

Consequently, a reduction of the kinetic energy along the caxis and along the diagonal in the $\mathrm{CuO}_{2}$ planes arises. In addition, both hopping elements experience an isotope effect since $\gamma$ is isotope dependent. We have excluded the renormalizations of $t_{1}$ and $t_{3}$, since the isotope effect arising from $t_{1}$ has the wrong doping dependence, whereas the third-nearest neighbor hopping does not contribute to it at all $[18,22]$. From the role played by $t_{2}$ and $t_{4}$ we conclude that a Jahn-Teller $\mathrm{Q}_{2}$-type lattice displacement together with a caxis mode is relevant for understanding the isotope effects, which will be shown subsequently in the experimental details. Another important aspect to be mentioned is the rigid band shift $\Delta^{*}$. This gives rise to density-density interband interactions which support two-band superconductivity [18, $22,24,25]$. The renormalization of the lattice degrees of freedom through the transformation leads to a rigid harmonic oscillator shift such that the pure lattice Hamiltonian can be written as

$\tilde{H}=\sum_{q, j} \hbar \tilde{\omega}_{q, j}\left(\tilde{b}_{q, j}^{+} \tilde{b}_{q, j}+\frac{1}{2}\right)$,

where the transformed frequencies are given by

$\tilde{\omega}_{q, j}^{2}=\omega_{q, j}^{(0) 2}-\gamma_{i}^{2}(q) \sum_{k} \frac{1}{\varepsilon(k)} \tanh \frac{\varepsilon(k)}{k T}$.

As is obvious from (7), a local lattice instability takes place if $\tilde{\omega}_{q, j}^{2} \rightarrow 0$. This is induced through the temperature dependence of the charge bath. It has to be noted, however, that we are not dealing with a long wave-length instability where momentum is relevant but with a local one, i.e., $q$ being finite, where real space comes into play. The transition to a modulated dynamic structure, which we identify with the pseudogap phase, is given by the implicit relation

$\omega_{q, j}^{(0) 2} / \gamma_{i}^{2}(q)=\sum_{k} \frac{1}{\varepsilon(k)} \tanh \frac{\varepsilon(k)}{k_{B} T^{*}}$.

Since both $\omega_{q, j}^{(0)}$ and $\gamma$ carry an isotope effect, $T^{*}$ becomes isotope dependent as well. This isotope effect is extensive and sign reversed as compared to the one on $T_{c}$, and is in agreement with experimental data [5,6]. Besides of the above mentioned superstructure formation, an important consequence of our approach appears in the local mean square displacement which is derived from (7) like:

$\sigma^{2}(T)=\hbar /\left(M \tilde{\omega}_{q, j}\right) \operatorname{coth}\left(\hbar \tilde{\omega}_{q, j} / 2 k T\right)$. 
Fig. 1 Temperature

dependence of the mean square displacement for an s-wave superconductor (filled circles) and a d-wave superconductor (open squares). The inset shows the experimental data from Ref. [27] (after Ref. [20])

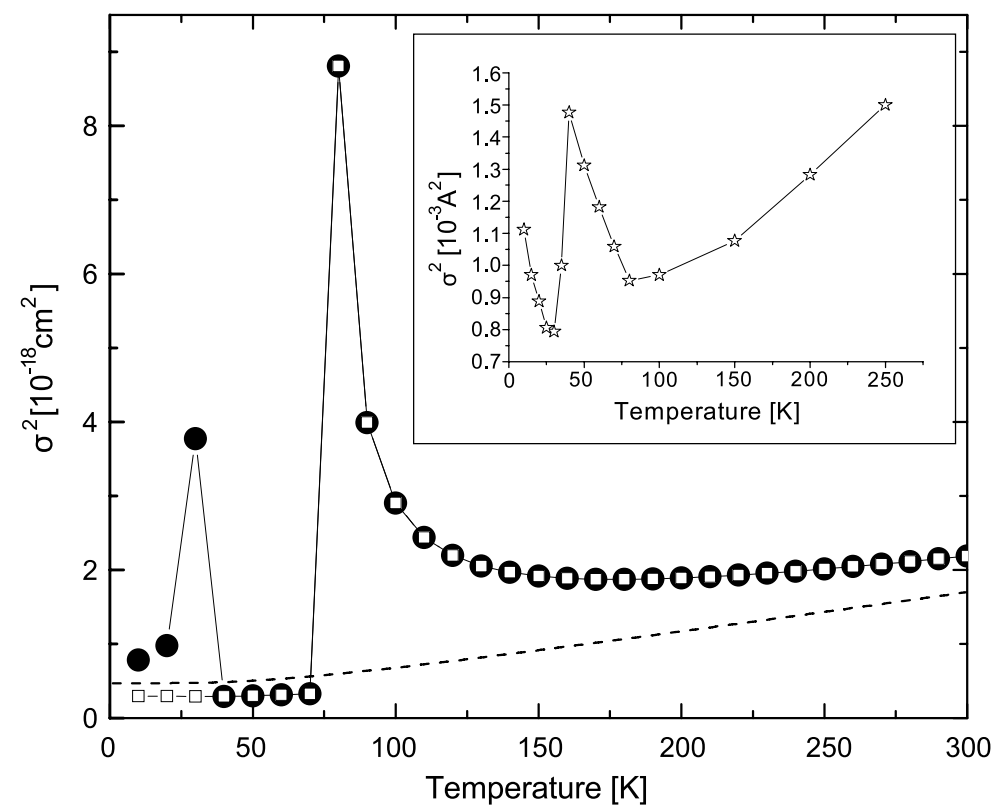

Since here the renormalized mode enters, an unusual upturn in $\sigma^{2}(T)$ appears at $T^{*}$ caused by the mode softening. In addition, superconductivity affects strongly the mean square displacement since at the onset of superconductivity $\tilde{\varepsilon}(k)$ has to be replaced by $E(k)=\sqrt{\tilde{\varepsilon}^{2}(k)+\Delta_{0}^{2}(k)}$, with $\Delta_{0}$ being the superconducting energy gap, where the superconducting gap symmetry can be either s-wave, d-wave or $(\mathrm{s}+\mathrm{d})$-wave. The results for $\sigma^{2}(T)$ are shown in Fig. 1 where the pairing symmetry in one case is of s-wave symmetry (filled circles) whereas in the other case it is of d-wave symmetry (open squares). Intermediate mixed pairing symmetries will be discussed in a forthcoming paper [26].

From Fig. 1 it is obvious that a sharp upturn in $\sigma^{2}(T)$ sets in at the onset of the pseudogap phase. Below $T^{*}$, fast dynamics dominate which reduce $\sigma^{2}(T)$ substantially. At $T_{c}$, a very different behavior is observed for the two pairing symmetries considered: while for d-wave pairing no anomaly in $\sigma^{2}(T)$ is apparent at $T_{c}$, the s-wave symmetry case exhibits a substantial influence on it signaled by a peak in the mean square displacement [20].

This finding is in agreement with experimental data which are shown as inset to Fig. 1 [27]. We conclude from the comparison between experiment and theory that EXAFS is an excellent tool to determine the pairing symmetry in polaronic superconductors.

While the role of polarons for the electronic energies has been discussed in a variety of early and recent work [28, 29], the consequences for the lattice degrees of freedom have so far not been considered in detail. Our approach thus provides a complementary view of both lattice and charge renormalizations caused by polaron formation. In a recent review, early developments in this field have been summarized [30] and updated recently in Ref. [31].

\section{Experimental Results}

In the following, the experimental results are presented which support the above theoretical modeling.

\subsection{Isotope Effects}

The oxygen-isotope $\left({ }^{16} \mathrm{O} /{ }^{18} \mathrm{O}\right)$ effect (OIE) on $T_{c}$ in the HTS was studied in detail already shortly after the discovery of high-temperature superconductivity [1]. The OIE on $T_{c}$ can be well described by the OIE exponent $\alpha_{\mathrm{O}}=$ $-d \ln T_{c} / d \ln M_{\mathrm{O}}$, where $M_{\mathrm{O}}$ denotes the oxygen ion mass. It was found that for all cuprate HTS families the OIE exponent $\alpha_{\mathrm{O}}$ shows a generic trend: In the underdoped regime, $\alpha_{\mathrm{O}}$ is large (even exceeding the BCS value of $\alpha_{\mathrm{O}}=0.5$ ) and becomes vanishingly small close to optimal doping [1-4]. An example of this generic behavior of $\alpha_{\mathrm{O}}$ as a function of $T_{c}$, together with theoretical calculations using an extended version of the above model, is shown in Fig. 2. Note that -as outlined above- the theoretical data (solid line) have been obtained by renormalizing $t_{2}$ and $t_{4}$, whereas the renormalization of $t_{1}$ (dashed line) yields the wrong doping dependence of $\alpha_{\mathrm{O}}$. In addition to this study, also site-selective OIE investigations of $\mathrm{Y}_{1-x} \mathrm{Pr}_{x} \mathrm{Ba}_{2} \mathrm{Cu}_{3} \mathrm{O}_{7-\delta}$ have been performed, thereby clearly revealing that the planar oxygen atoms contribute dominantly to the total OIE on $\mathrm{T}_{c}$ at all doping levels, whereas the contribution of chain and apical oxygen ions is only negligible [32-34]. However, these findings do not imply that chain and apical oxygen ions are irrelevant to superconductivity, since their small density of states as compared to the one of the planar oxygen ions leads to a small or even vanishing contribution to the total OIE [35]. 


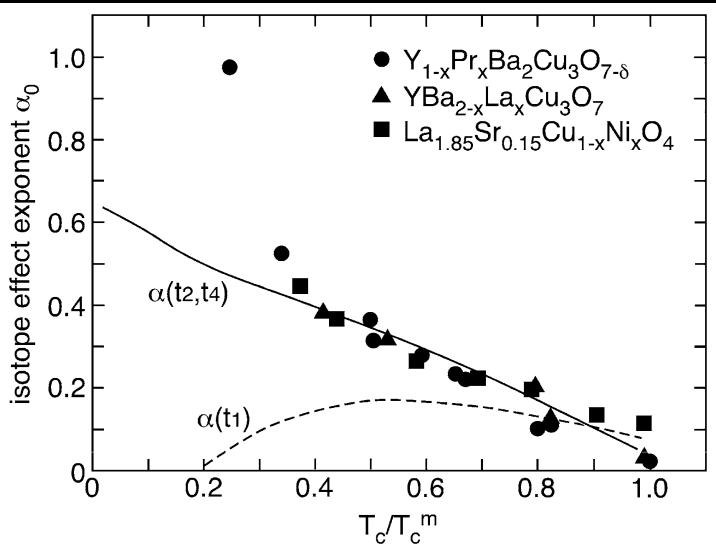

Fig. 2 The oxygen isotope exponent $\alpha_{\mathrm{O}}$ as a function of $T_{c} / T_{c}^{m}$ for various cuprate families ( $T_{c}^{m}$ denotes the maximum $T_{c}$ for a particular family). The dashed line refers to the calculated $\alpha_{\mathrm{O}}$ when only the nearest neighbor hopping integral $\left(t_{1}\right)$ is renormalized; the solid line is theoretically derived where both second-nearest neighbor and interplanar hopping integrals $\left(t_{2}, t_{4}\right)$ are renormalized. Black circles, black squares, and black triangles represent experimental data (after Refs. [18, 22])

The OIE on the transition temperatures of the various phases observed in all cuprate HTS [superconducting (SC), spin-glass (SG), and antiferromagnetic (AFM)] were investigated for the prototype system of cuprates $\mathrm{Y}_{1-x}$ $\mathrm{Pr}_{x} \mathrm{Ba}_{2} \mathrm{Cu}_{3} \mathrm{O}_{7}$ (see Fig. 3) [7]. All transition temperatures $T_{c}, T_{N}$, and $T_{g}$ exhibit an OIE which is strongest where the respective phase terminates. In addition, the OIE on the magnetic transition temperatures $T_{g}$ (SG) and $T_{N}$ (AFM) are sign reversed as compared to the ones on $T_{c}$. In the coexistence regime of the SG and SC phase, a two-component behavior is observed, where the isotope-induced decrease of $T_{c}$ in the SC region leads to a corresponding increase of $T_{g}$ in the $\mathrm{SG}$ region. This observation suggests that in this regime a phase separation sets in, where the superfluid density coexists with a related SG regime. Since the OIE on $T_{c}$ can be accounted for by polaron formation [18, 22, 36], the one on $T_{g}$ is very likely dominated by the same physics. By relating $T_{N}$ to the metal insulator transition, a reduction in kinetic energy caused by polaron formation explains this unconventional OIE as well. Further support for the importance of lattice effects and in particular for the polaron model discussed above is due to the observation of the extensive OIE on the pseudogap temperature $T^{*}$. XANES experiments in $\mathrm{La}_{2-x} \mathrm{Sr}_{x} \mathrm{CuO}_{4}$ [5], as well as inelastic neutron scattering studies of $\mathrm{HoBa}_{2} \mathrm{Cu}_{4} \mathrm{O}_{8}$ and $\mathrm{La}_{1.81} \mathrm{Sr}_{0.18} \mathrm{Ho}_{0.04} \mathrm{CuO}_{4}$ [6], revealed an extremely large and sign-reversed OIE on $T^{*}$. Furthermore, from the neutron scattering experiments, even a larger ${ }^{63} \mathrm{Cu} /{ }^{65} \mathrm{Cu}$ isotope effect on $T^{*}$ was observed in $\mathrm{HoBa}_{2} \mathrm{Cu}_{4} \mathrm{O}_{8}$, which is absent in $\mathrm{La}_{1.81} \mathrm{Sr}_{0.18} \mathrm{Ho}_{0.04} \mathrm{CuO}_{4}$ [6].

This suggests that an umbrella-type mode is responsible for the formation of the pseudogap in $\mathrm{HoBa}_{2} \mathrm{Cu}_{4} \mathrm{O}_{8}$, which

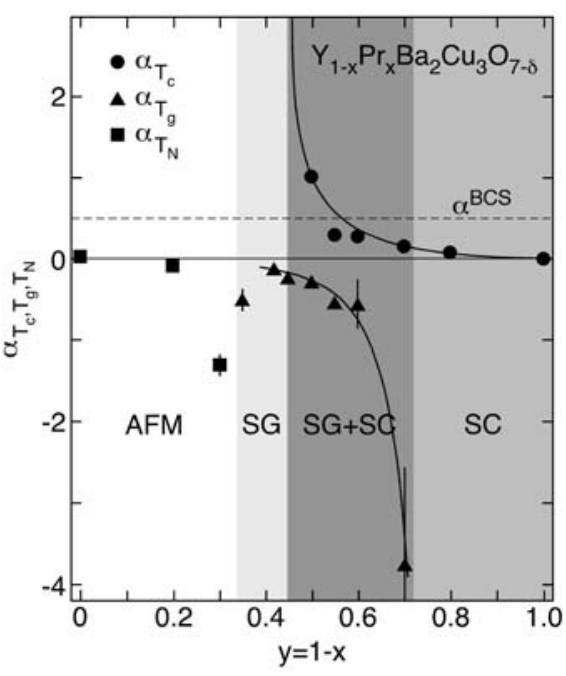

Fig. 3 OIE exponents $\alpha_{\mathrm{O}}$ of $T_{c}, T_{g}$, and $T_{\mathrm{N}}$ for ${ }^{16} \mathrm{O} /{ }^{18} \mathrm{O}$-substituted $\mathrm{Y}_{1-x} \mathrm{Pr}_{x} \mathrm{Ba}_{2} \mathrm{Cu}_{3} \mathrm{O}_{7}$ as a function of the Pr content $y=1-x$. The dashed line corresponds to the BCS value $\alpha_{\mathrm{O}}^{\mathrm{BCS}}=0.5$. The solid lines are guides to the eye. The meaning of the areas denoted by AFM, SG, $\mathrm{SG}+\mathrm{SC}$, and SC is explained in the text (after Ref. [7])

is absent in $\mathrm{La}_{1.81} \mathrm{Sr}_{0.18} \mathrm{Ho}_{0.04} \mathrm{CuO}_{4}$ having no apical oxygen. These extensive isotope effects may be explained in terms of dynamical charge ordering. Evidently, the polaron picture discussed above implies that also ordering in the spin system occurs at $T^{*}$ as observed in $\mathrm{YBa}_{2} \mathrm{Cu}_{4} \mathrm{O}_{8}$ by means of NQR [37]. This is consistent with the almost vanishing OIE on $T^{*}$ (comparable to the OIE on $T_{c}$ ) detected by NQR since NQR is mainly sensitive to the spin system [37].

An unexpected OIE on the zero-temperature in-plane magnetic penetration depth $\lambda_{a b}(0)$ was observed in several families of HTS [2, 38-40], which is not expected for conventional superconductors where the electronic and vibronic degrees of freedom are decoupled due to the extensive mass difference between ions and electrons (Migdal theorem) [41]. A breakdown of this theorem may, however, occur if the carriers are strongly coupled to the lattice as in the case of polarons. This implies that the effective carrier mass $m^{*}$ can no longer be decoupled from the ionic mass $M$, giving rise to an isotope effect on the magnetic penetration depth. Detailed OIE studies of $\lambda_{a b}(0)$ were performed for various families of HTS at different doping levels by means of various experimental techniques [2, 38-40]. The unexpected interesting result is that the OIE on $\lambda_{a b}(0)$ exhibits almost the same generic trend with doping as the one on $T_{c}$ (see Fig. 4). At low doping levels, a linear correlation between both OIEs is found. However, near optimal doping, a deviation from this linear behavior sets in. While the linear correlation at low doping is well explained by the polaron model [18, 22, 36] described above (straight line in Fig. 3), this model cannot account for the deviations near the optimal doping. This is most likely due to the fact that the 


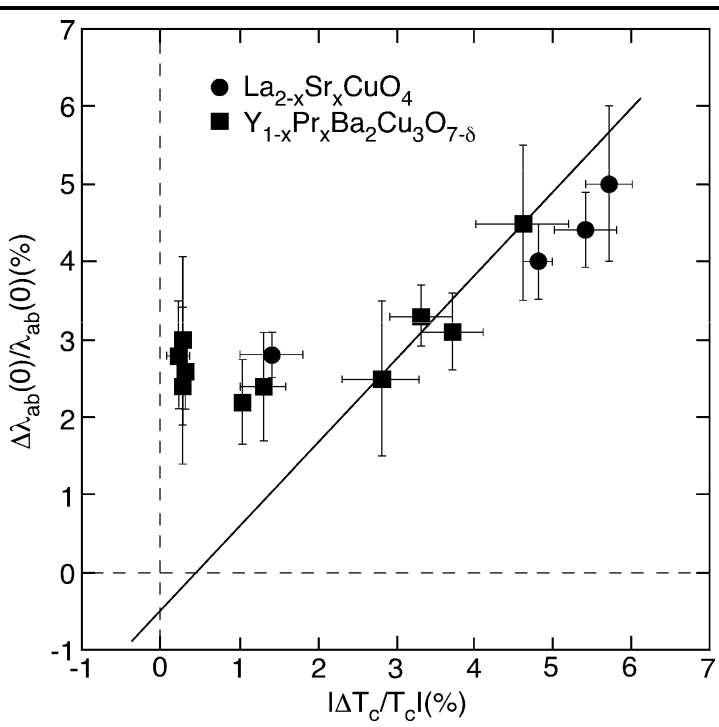

Fig. 4 Plot of the OIE shift $\Delta \lambda_{a b}(0) / \lambda_{a b}(0)$ vs. the OIE shift $\left|\Delta T_{c} / T_{c}\right|$ for $\mathrm{La}_{2-x} \mathrm{Sr}_{x} \mathrm{CuO}_{4}$ and $\mathrm{Pr}_{x} \mathrm{Y}_{1-x} \mathrm{Ba}_{2} \mathrm{Cu}_{3} \mathrm{O}_{7-\delta}$ using different experimental techniques and types of samples. The data are summarized in Table 2 of Ref. [2]. The solid line corresponds to model calculations of the OIE on $\Delta \lambda_{a b}(0) / \lambda_{a b}(0)$ obtained from the theoretical results for the average gap $[18,22,36]$. Note that a negative OIE on the average gap is predicted at optimal doping and for overdoped systems [18] (after Refs. [18, 22, 36])

polaron coupling is doping dependent, which has not been taken into account yet.

Furthermore, this polaron model [18, 22, 36], also predicts a doping-dependent isotope effect on the zerotemperature superconducting gap $\Delta_{0}$ which was recently observed experimentally [8]. In conventional superconductors the isotope effect on $\Delta_{0}$ is simply determined through the isotope effect on $T_{c}$ via the relation $2 \Delta_{0} / k_{B} T_{c} \approx 3.52$. However, in the HTS this relation does not necessarily hold, because $T_{c}$ strongly depends on doping. Moreover, the cuprate HTS have a complex superconducting order parameter where $(\mathrm{s}+\mathrm{d})$-wave symmetries are mixed as will be shown below. A systematic study of the OIE on $\Delta_{0}$ in $\mathrm{Y}_{1-x} \mathrm{Pr}_{x} \mathrm{Ba}_{2} \mathrm{Cu}_{3} \mathrm{O}_{7-\delta}$ for various doping levels $x$ was performed by means of SQUID magnetization experiments [8]. The values of $\Delta_{0}$ extracted from the temperature dependence of the superfluid density are proportional to $T_{C}$ with $2 \Delta_{0} / k_{B} T_{c} \approx 5.74$. This ratio, which was predicted theoretically $[18,22,36]$, is substantially larger than the BCS value. In addition, the OIE on $\Delta_{0}$ scales linearly with the one on $T_{c}$, exhibiting a sign reversal of the OIE on $\Delta_{0}$ and $T_{c}$ near optimal doping in agreement with model predictions $[18,22,36]$.

The various OIE reported here clearly demonstrate that lattice effects play an important role in all phases of the HTS and support the idea that polaron formation is the cause of all these isotope effects.

\subsection{Mixed Order Parameter Symmetries}

The order parameter in cuprate HTS is mostly assumed to be of d-wave symmetry due to the presence of the $\mathrm{CuO}_{2}$ planes which are essential for the occurrence of superconductivity in the HTS. Even though either static or dynamic distortion of the $\mathrm{CuO}_{2}$ planes destroys the cubic symmetry of the planes, most theoretical models ignore the observed orthorhombicity and idealize the planar structure. Already more than ten years ago, however, it was emphasized that the cuprate HTS must have a more complex order parameter $(d+s, d+$ is, etc. $)[42,43]$. This idea was in part pursued because two gaps have been observed in Nbdoped $\mathrm{SrTiO}_{3}$ [44], the first oxide in which superconductivity was detected. Indeed, from various experiments it has been concluded that a complex order parameter is realized in the cuprate HTS, including nuclear magnetic resonance (NMR) [42], Raman scattering [45, 46], and neutron crystalfield spectroscopy [47].

In order to probe the order parameter symmetry, bulk sensitive experiments on single crystals are required. Muonspin rotation $(\mu \mathrm{SR})$ was demonstrated to be a unique tool to investigate the superfluid density in the bulk of a superconductor. Recently, $\mu \mathrm{SR}$ was used to determine the temperature dependence of the superfluid density along the three crystallographic axes in single crystals of the cuprate HTS, from which information on the gap symmetry can be extracted [13-15]. The experiments were carried out on three different families of the cuprate HTS, namely $\mathrm{La}_{2}{ }_{x} \mathrm{Sr}_{x} \mathrm{CuO}_{4}, \mathrm{YBa}_{2} \mathrm{Cu}_{4} \mathrm{O}_{8}$, and $\mathrm{YBa}_{2} \mathrm{Cu}_{3} \mathrm{O}_{7-\delta}$. In all three systems a pronounced inflection point in the in-plane superfluid density was observed at low temperatures (the results for $\mathrm{YBa}_{2} \mathrm{Cu}_{4} \mathrm{O}_{8}$ and $\mathrm{YBa}_{2} \mathrm{Cu}_{3} \mathrm{O}_{7-\delta}$ are shown in Fig. 5), which evidences the coexistence of a small and a large gap. From the magnetic field dependence of the superfluid density it is concluded [49] that in the $\mathrm{CuO}_{2}$ planes a small s-wave gap coexists with a dominant d-wave gap. However, as is shown in Fig. 5, the temperature dependence of the superfluid density along the c-axis differs considerably from those along $a$ and $b$ directions. It shows no inflection point here and saturates at low temperatures (compare $\sigma_{a}$ and $\sigma_{b}$ with $\sigma_{c}$ in Fig. 5). This is characteristic for a pure s-wave gap. From a theoretical point of view, a mixed order parameter scenario requires [18, 22, 48] that also a small d-wave admixture is present. However, the d-wave component appears to be too small to be observed experimentally $[14,15]$. A similar admixture of a 20-25\% s-wave component to the d-wave gap symmetry was also observed for $\mathrm{HoBa}_{2} \mathrm{Cu}_{3} \mathrm{O}_{6.56}, \mathrm{HoBa}_{2} \mathrm{Cu}_{4} \mathrm{O}_{8}$, and $\mathrm{La}_{1.81} \mathrm{Sr}_{0.15} \mathrm{Ho}_{0.04} \mathrm{CuO}_{4}$ by means of inelastic neutron scattering [47]. Furthermore, it should be pointed out that also c-axis tunnelling in $\mathrm{YBa}_{2} \mathrm{Cu}_{3} \mathrm{O}_{7-\delta}$ [49] and bicrystal-twist Josephson junction experiments in $\mathrm{Bi}_{2} \mathrm{Sr}_{2} \mathrm{CaCu}_{2} \mathrm{O}_{8+\delta}$ [50] 
Fig. 5 a) Temperature dependences of the $\mu \mathrm{SR}$ relaxation rate $\sigma_{a} \propto \lambda_{a}^{-2}$, $\sigma_{b} \propto \lambda_{b}^{-2}$, and $\sigma_{c} \propto \lambda_{c}^{-2}$ in single crystals of $\mathrm{YBa}_{2} \mathrm{Cu}_{4} \mathrm{O}_{8}$ measured along the three principal crystallographic axes $a, b, c$. The full lines are obtained as outlined in Refs. [13-15, 48] (after Ref. [15]). b) Temperature dependence of the $\mu \mathrm{SR}$ relaxation rate $\sigma_{a} \propto \lambda_{a}^{-2}, \sigma_{b} \propto \lambda_{b}^{-2}$, and $\sigma_{c} \propto \lambda_{c}^{-2}$ in single crystals of $\mathrm{YBa}_{2} \mathrm{Cu}_{3} \mathrm{O}_{7-\delta}$ measured along the three principal crystallographic axes $a, b, c$. The lines are results from model calculations as discussed in Refs. [13-15, 48] (after Ref. [14])

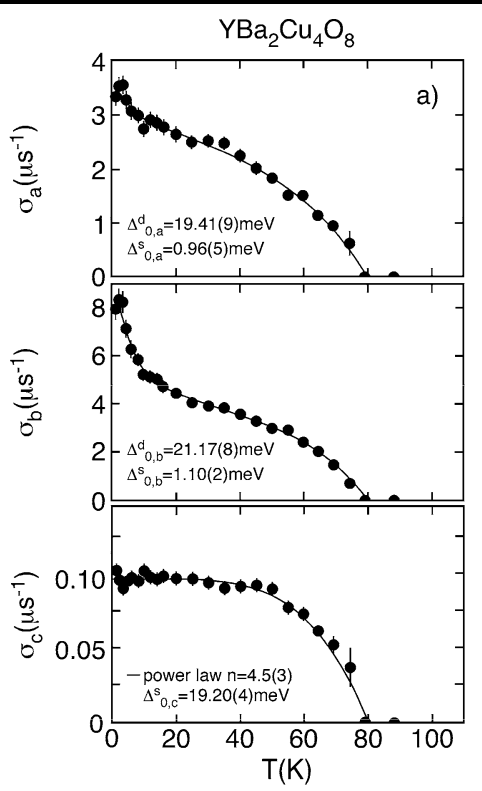

provide evidence for a dominant s-wave order parameter along the c-axis direction, in agreement with indirect optical pulsed probe experiments in $\mathrm{YBa}_{2} \mathrm{Cu}_{3} \mathrm{O}_{7-\delta}$ [51].

The peculiar behavior of the temperature dependence of the superfluid density observed in all three cuprate systems investigated, strongly suggests that the observed mixed order parameter symmetries are intrinsic and generic to all the cuprate HTS. In particular, the finding that the gap along the c-axis has a predominant s-wave character demonstrates the importance of the coupling between the $\mathrm{CuO}_{2}$ planes, an experimental fact which in most theoretical models is completely neglected.

\section{Conclusions}

We have presented compelling theoretical and experimental evidence that lattice involvement in all phases of the HTS is present in terms of polaron formation. These polarons are of intermediate size and local, which means that a dynamical structuring of superlattice type takes place where two components are formed. These components are the cause of coexisting complex order parameters, namely $\mathrm{s}+\mathrm{d}$, with a predominantly s-wave symmetry along the c-axis. The observed isotope effects are a natural consequence of the modeling. They point to the important role of a $\mathrm{Q}_{2}$-type Jahn-Teller mode. The subtle local lattice effects emerge in real space pairing with short coherence lengths, in strong contrast to BCS phonon-induced pairing where long wavelength phonons are the mediator and long coherence lengths the consequence.

Acknowledgements It is a pleasure to acknowledge many useful discussions with K.A. Müller, R. Khasanov, A.R. Bishop, A. Simon,
A. Bianconi, H. Oyanagi, and N.L. Saini. Financial support is acknowledged from the Swiss National Science Foundation, the K. Alex Müller Foundation, the European program CoMePhS, and the NCCR MaNEP.

\section{References}

1. Franck, J.P.: In: Ginsberg, D.M. (ed.) Physical Properties of High Temperature Superconductors, p. 189. World Scientific, Singapore (1994)

2. Keller, H.: In: Müller, K.A., Bussmann-Holder, A. (eds.) Superconductivity in Complex Systems. Springer Series Structure and Bonding, vol. 114, p. 143. Springer, Berlin (2005), and references therein

3. Zhao, G.M., Conder, K., Keller, H., Müller, K.A.: J. Phys.: Cond. Mat. 10, 9055 (1998)

4. Zhao, G.M., Keller, H., Conder, K.: J. Phys.: Condens. Matter 13, R569 (2001)

5. Bianconi, A.: In: Müller, K.A., Bussmann-Holder, A. (eds.) Superconductivity in Complex Systems. Springer Series Structure and Bonding, vol. 114, p. 287. Springer, Berlin (2005)

6. Furrer, A.: In: Müller, K.A., Bussmann-Holder, A. (eds.) Superconductivity in Complex Systems. Springer Series Structure and Bonding, vol. 171, p. 171. Springer, Berlin (2005), and references therein

7. Khasanov, R., Shengelaya, A., Di Castro, D., Morenzoni, E., Maisuradze, A., Savić, I.M., Conder, K., Pomjakushina, E., Bussmann-Holder, A., Keller, H.: Phys. Rev. Lett. 101, 077001 (2008)

8. Khasanov, R., Strässle, S., Conder, K., Pomjakushina, E., Bussmann-Holder, A., Keller, H.: Phys. Rev. B 77, 104530 (2007)

9. Bianconi, A., Saini, N.L., Lanzara, A., Missori, M., Rossetti, T., Oyanagi, H., Yamaguchi, H., Oka, K., Ito, T.: Phys. Rev. Lett. 76, 3412 (1996)

10. Saini, N.L., Lanzara, A., Oyanagi, H., Yamaguchi, H., Oka, K., Ito, T., Bianconi, A.: Phys. Rev. B 55, 12759 (1997)

11. Oyanagi, H., Tsukuda, A., Naito, M., Saini, N.L.: Phys. Rev. B 75, 024511 (2007)

12. Sharma, R.P., Ogale, S.B., Zhang, Z.H., Liu, J.R., Chu, W.K., Veal, B., Paulakis, A., Zheng, H., Ventakesan, T.: Nature (Lond.) 404, 736 (2000) 
13. Khasanov, R., Shengelaya, A., Maisuradze, A., La Mattina, F., Bussmann-Holder, A., Keller, H., Müller, K.A.: Phys. Rev. Lett. 98, 057007 (2007)

14. Khasanov, R., Strässle, S., Di Castro, D., Masui, T., Miyasaka, S., Tajima, S., Bussmann-Holder, A., Keller, H.: Phys. Rev. Lett. 99, 237601 (2007)

15. Khasanov, R., Shengelaya, A., Karpinski, J., Bussmann-Holder, A., Keller, H., Müller, K.A.: J. Supercond. Novel Magn. 21, 81 (2008)

16. Bednorz, J.G., Müller, K.A.: Z. Phys. B 64, 189 (1986)

17. Bednorz, J.G., Müller, K.A.: Adv. Chemie 100, 757 (1988), Nobel Lecture

18. Bussmann-Holder, A., Keller, H.: In: Alexandrov, A.S. (ed.) Polarons in Advanced Matter. Springer Series in Material Science, vol. 103, p. 599. Springer, Berlin (2007)

19. Bussmann-Holder, A., Müller, K.A., Micnas, R., Büttner, H., Simon, A., Bishop, A.R.: J. Phys.: Cond. Mat. 13, L169 (2001).

20. Bussmann-Holder, A., Keller, H., Bishop, A.R., Simon, A., Müller, K.A., J. Supercond. Nov. Magn., published online 15 Oct. 2008

21. Lang, S.G., Firsov, Yu.A.: Sov. Phys. JETP 16, 1302 (1963)

22. Bussmann-Holder, A., Keller, H.: Eur. Phys. J. 44, 487 (2005)

23. Pavarini, E., Dasgupta, I., Saha-Dasgupta, T., Jepsen, O., Andersen, O.K.: Phys. Rev. Lett. 87, 047003 (2001)

24. Bussmann-Holder, A., Keller, H., Müller, K.A.: In: Müller, K.A., Bussmann-Holder, A. (eds.) Superconductivity in Complex Systems. Springer Series Structure and Bonding, vol. 114, p. 365. Springer, Berlin (2005)

25. Bussmann-Holder, A., Bishop, A.R., Büttner, H., Egami, T., Micnas, R., Müller, K.A.: J. Phys.: Cond. Mat. 13, L545 (2001)

26. Bussmann-Holder, A., Keller, H., Müller, K.A.: to be published

27. Oyanagi, H., Zhang, C., Tsukada, A., Naito, M.: J. Phys. Conf. Ser. 108, 012038 (2008)

28. Kresin, V.Z., Ovchinnikov, Y.N., Wolf, S.A.: Phys. Rep. 431, 231 (2006)

29. Alexandrov, A.S. (ed.): Polarons in Advanced Matter. Springer Series in Material Science, vol. 103, p. 257. Springer, Berlin (2007)

30. Müller, K.A.: J. Phys.: Cond. Mat. 19, 251002 (2007)

31. Keller, H., Bussmann-Holder, A., Müller, K.A.: Mater. Today 11, 38 (2008)

32. Zech, D., Keller, H., Conder, K., Kaldis, E., Liarokapis, E., Poulakis, N., Müller, K.A.: Nature (Lond.) 371, 681 (1994)
33. Zhao, G.M., Ager III, J.W., Morris, D.E.: Phys. Rev. B 54, 14982 (1996)

34. Khasanov, R., Shengelaya, A., Morenzoni, E., Angst, M., Conder, K., Savić, I.M., Lampakis, D., Liarokapis, E., Tatsi, A., Keller, H.: Phys. Rev. B 68, R220506 (2003).

35. Bussmann-Holder, A., Bishop, A.R., Genzel, L., Simon, A.: Phys. Rev. B 55, 11751 (1997)

36. Bussmann-Holder, A., Keller, H., Bishop, A.R., Simon, A., Micnas, R., Müller, K.A.: Europhys. Lett. 72, 423 (2005)

37. Raffa, F., Ohno, T., Mali, M., Roos, J., Brinkmann, D., Conder, K., Eremin, M.: Phys. Rev. Lett. 81, 5912 (1998)

38. Zhao, G.M., Hunt, M.B., Keller, H., Müller, K.A.: Nature (Lond.) 385, 236 (1997)

39. Hofer, J., Conder, K., Sasagawa, T., Zhao, G.M., Willemin, M., Keller, H., Kishio, K.: Phys. Rev. Lett. 84, 4192 (2000)

40. Khasanov, R., Eshchenko, D.G., Luethens, H., Morenzoni, E., Prokscha, T., Suter, A., Garifianov, N., Mali, M., Roos, J., Conder, K., Keller, H.: Phys. Rev. Lett. 92, 057602 (2004)

41. Migdal, B.: Sov. Phys. JETP 7, 996 (1958)

42. Müller, K.A., Keller, H.: In: High $T_{c}$ Superconductivity: 10 Years after the Discovery. NATO ASI Ser. E, vol. 343, pp. 7. Kluwer Acad., Amsterdam (1997)

43. Müller, K.A.: Nature (Lond.) 377, 133 (1995)

44. Binnig, G., Baratoff, A., Hoenig, H.E., Bednorz, J.G.: Phys. Rev. Lett. 45, 1352 (1980)

45. Cardona, M., Liu, R., Thomsen, C., Kress, W., Schönherr, E., Bauer, M., Genzel, L., König, W.: Solid State Comm. 67, 789 (1988)

46. Masui, T., Limonov, M., Uchiyama, H., Lee, S., Tajima, S.: Phys. Rev. B 68, 060506 (2003)

47. Furrer, A.: J. Supercond. Novel Magn. 21, 1 (2008)

48. Bussmann-Holder, A., Khasanov, R., Shengelaya, A., Maisuradze, A., La Mattina, F., Keller, H., Müller, K.A.: Europhys. Lett. 77, 27002 (2007)

49. Sun, A.G., Gajewski, D.A., Maple, M.P., Dynes, R.C.: Phys. Rev. Lett. 72, 2267 (1994)

50. Li, Q., Tsay, Y.N., Suenaga, M., Klemm, R.A., Gu, G.D., Koshizuka, N.: Phys. Rev. Lett. 83, 4160 (1999)

51. Kabanov, V.V., Demsar, J., Podobnik, B., Mihailovic, D.: Phys. Rev. B 59, 1497 (1999) 